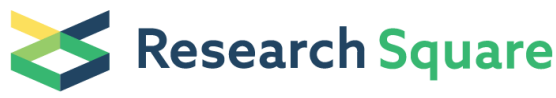 \\ Preprints are preliminary reports that have not undergone peer review. \\ They should not be considered conclusive, used to inform clinical practice, or referenced by the media as validated information.
}

\section{Increased uptake of 68Ga-DOTA-FAPI-04 in bones and joints: metastases and beyond}

\section{Chunxia Qin}

Union Hospital of Tongji Medical College of Huazhong University of Science and Technology

\section{Yangmeihui Song}

Union Hospital of Tongji Medical College of Huazhong University of Science and Technology

\section{Xi Liu}

Union Hospital of Tongji Medical College of Huazhong University of Science and Technology

\section{Yongkang Gai}

Union Hospital of Tongji Medical College of Huazhong University of Science and Technology

\section{Qingyao Liu}

Union Hospital of Tongji Medical College of Huazhong University of Science and Technology

\section{Weiwei Ruan}

Union Hospital of Tongji Medical College of Huazhong University of Science and Technology

\section{Fang Liu}

Union Hospital of Tongji Medical College of Huazhong University of Science and Technology

\section{Fan $\mathrm{Hu}$}

Union Hospital of Tongji Medical College of Huazhong University of Science and Technology

Xiaoli Lan ( $\nabla$ xiaoli_lan@hust.edu.cn )

Union Hospital, Tongji Medical College, Huazhong University of Science \&amp; Technology https://orcid.org/0000-00027263-7399

\section{Research Article}

Keywords: PET, fibroblast activation protein inhibitor, differential diagnosis, bone metastases, osteofibrous dysplasia

Posted Date: April 7th, 2021

DOI: https://doi.org/10.21203/rs.3.rs-395267/v1

License: (9) (i) This work is licensed under a Creative Commons Attribution 4.0 International License. Read Full License

Version of Record: A version of this preprint was published at European Journal of Nuclear Medicine and Molecular Imaging on July 9th, 2021. See the published version at https://doi.org/10.1007/s00259-021-05472-3. 


\section{Abstract}

Purpose: To describe the uptake of ${ }^{68} \mathrm{Gallium}$-labelled fibroblast activation protein inhibitor $\left({ }^{68} \mathrm{Ga}-\mathrm{FAPI}\right)$ in bones and joints for better understanding of the role of ${ }^{68} \mathrm{Ga}-\mathrm{FAPI}$ PET in benign and malignant bone lesions and joint diseases.

Methods: All $129{ }^{68}$ Ga-FAPI PET/MR or PET/CT scans from June 1, 2020 to February 20, 2021 performed at our PET centre were retrospectively reviewed. Foci of elevated ${ }^{68} \mathrm{Ga}$-FAPI uptake in bones and joints were identified. All lesions were divided into malignant and benign disease. Benign lesions included osteofibrous dysplasia, periodontitis, degenerative bone diseases, arthritis, and other inflammatory or trauma-related abnormalities. The number, locations and SUVmax of all lesions were recorded and analysed.

Results: Elevated uptake of ${ }^{68} \mathrm{Ga}$-FAPI in/around bones and joints were found in 82 cases (63.57\%). A total of 295 lesions were identified, including 94 (31.9\%) malignant lesions (all were metastases) and 201 (68.1\%) benign lesions. The benign lesions consisted of 13 osteofibrous dysplasia, 48 degenerative bone disease, 33 periodontitis, 56 arthritis, and 51 other inflammatory or trauma-related abnormalities. Spine, shoulder joint, alveolar ridge, and pelvis were the most commonly involved locations. Bone metastases were mainly distributed in the spine, pelvis and ribs. Among benign diseases, periodontitis and arthritis are site-specific. The mean SUVmax of bone metastases was significantly higher than that of benign diseases (7.14 \pm 4.33 vs. $3.57 \pm 1.60, p<0.0001)$, but overlap existed. The differences in SUVmax among subgroups of benign diseases were statistically significant $(p<0.0001)$, with much higher uptake in periodontitis $(4.45 \pm 1.17)$.

Conclusion: ${ }^{68} \mathrm{Ga}$-FAPI accumulated in both bone metastases and some benign diseases of bones and joints. Although the uptake of ${ }^{68} \mathrm{Ga}$-FAPI was often higher in bone metastases, this finding cannot be used to distinguish between benign and malignant lesions.

\section{Background}

Fibroblast activation protein (FAP) is overexpressed in cancer-associated fibroblasts in $>90 \%$ of epithelial tumors and in activated fibroblasts associated with remodelling of the extracellular matrix [1]. It is a promising target for the diagnosis of tumors and some non-malignant diseases [2, 3]. Various quinolone-based FAP inhibitors (FAPIs) radiolabelled with different radionuclides demonstrated specific binding to FAP [4-6]. Their clinical application has been of interest over the past two years $[7,8] .{ }^{68}$ Gallium-labeled FAPI ( $\left.{ }^{68} \mathrm{Ga}-\mathrm{FAPI}\right)$ positron emission tomography/computed tomography (PET/CT) has been used for imaging various types of cancers and outperforms ${ }^{18}$ F-FDG PET in cancers such as gastrointestinal cancers, cholangiocarcinoma, and hepatocellular cancer [9-11]. The potential of FAPI as a theranostic tool also warrants special attention $[12,13]$. However, ${ }^{68} \mathrm{Ga}$-FAPI uptake in benign tumors such as angiomyolipoma, and solitary fibrous tumors has been reported [14-16]. Uptake has also been identified in FAP-related non-neoplastic disease entities, including IgG4-related diseases, cardiovascular disease, and idiopathic retroperitoneal fibrosis [17-21]. These results render it a relatively nonspecific tumor agent.

Several studies have reported that ${ }^{68} \mathrm{Ga}$-FAPI outperformed ${ }^{18} \mathrm{~F}-\mathrm{FDG}$ PET/CT in identifying bone metastases [11, 22-24]. Two case reports revealed that intense ${ }^{68} \mathrm{Ga}-\mathrm{FAPI}$ uptake was observed in shoulder arthritis [25] and degenerative osteophyte [26]. Some preclinical studies have been reported high expression of FAP in bones and joints in arthritis and degenerative osteophytes [27-29]. To date, there are no data on the analysis of ${ }^{68} \mathrm{Ga}-\mathrm{FAPI}$ uptake in human bones and joints in large patient cohorts.

We retrospectively reviewed the images of all patients underwent ${ }^{68} \mathrm{Ga}-\mathrm{FAPI}$ PET/MRI or PET/CT in our PET centre, and reassessed ${ }^{68} \mathrm{Ga}$-FAPI accumulation in bones and joints to evaluate the uptake pattern of ${ }^{68} \mathrm{Ga}$-FAPI in malignant and benign bone and joint lesions to avoid misdiagnosis in current more and more application of ${ }^{68} \mathrm{Ga}$-FAPI PET in tumor diagnosis. 


\section{Methods}

\section{Patients}

We retrospectively reviewed the images of all patients who underwent ${ }^{68} \mathrm{Ga}$-FAPI PET/CT or PET/MR in our PET centre from June 1, 2020 to February 20, 2021. The patient cohort is a subgroup of two prospective trials focused on the diagnostic accuracy of ${ }^{68} \mathrm{Ga}$-FAPI PET in malignant tumors and fibrous diseases (ClinicalTrials.gov Identifier: NCT 04554719 and NCT 04605939). The study was approved by the Ethics Committee of Union Hospital, Tongji Medical College, Huazhong University of Science and Technology. All patients signed an informed consent before undergoing ${ }^{68}$ Ga-FAPI PET imaging.

\section{${ }^{68}$ Ga-FAPI PET/MR or PET/CT scanning}

${ }^{68} \mathrm{Ga}-\mathrm{FAPI}$ was synthesized according to a previously published method [11]. In brief, ${ }^{68} \mathrm{Gallium}\left({ }^{68} \mathrm{Ga}\right)$ that was eluted from a ${ }^{68} \mathrm{Ge} /{ }^{68} \mathrm{Ga}$ generator (ITG, Munich, Germany) was reacted with $25 \mu \mathrm{g}(28.6 \mathrm{nmol})$ of the DOTA-FAPI-04 ligand (CS Bio Company, Menlo Park CA, USA) in a manual synthesis module (ITG). The pH value was adjusted using $2.5 \mathrm{M}$ sodium acetate buffer. After heating to $100^{\circ} \mathrm{C}$ for 20 minutes, the product was purified and isolated by a solid-phase column (Sep-Pak, Waters Associates, Milford MA, USA). Quality control of the radiosynthesis was performed by high-performance liquid chromatography, and radiochemical purity exceeding $95 \%$ was accepted for injection. A total of $1.85-3.70 \mathrm{MBq}(0.05-0.10$ $\mathrm{mCi} / \mathrm{kg}{ }^{68} \mathrm{Ga}$-FAPI was administered intravenously.

$\mathrm{PET} / \mathrm{MR}$ or PET/CT scanning was performed $20-60$ min after ${ }^{68} \mathrm{Ga}-\mathrm{FAPI}$ administration. PET/MR was performed on a hybrid PET/MR scanner (3.0 T, SIGNA TOF-PET/MR, GE Healthcare, Milwaukee WI, USA). MR sequences included an axial highresolution T1-weighted fast spoiled gradient-echo sequence (LAVA Flex sequence) and T2-weighted (T2W) sequence from the skull base to the upper thighs, and diffusion-weighted sequences were acquired if needed. PET was acquired simultaneously in four to five bed positions with an acquisition time of 3 minutes per bed position (axial FOV: $25.8 \mathrm{~cm}$, matrix size: $344 \times$ 344). Images of the head were acquired separately. PET/CT (Discovery VCT, GE Healthcare) was performed in patients suspected of pulmonary fibrosis or with contraindications to MR. Both CT and PET were acquired from the skull vertex to the upper thighs with PET acquisition for 3 minutes per bed position. All images were reconstructed using a 3D attenuationweighted iterative ordered-subset expectation maximization algorithm with 3 iterations and 21 subsets.

\section{Image interpretation and analysis}

All images were reviewed using the AW workstation (AW4.6, GE Healthcare). Two nuclear medicine physicians (X. L., with 24 years of experience and C. Q., with 12 years of experience in nuclear medicine) and two radiologists (X. L., with 24 years of experience in orthopaedic radiology and F. L. with 20 years of experience in radiology and 5 years of experience in nuclear oncology) reviewed PET/MR and PET/CT images and conventional images (like X-ray, CT or MR). All reviewers analysed the images independently. Any differences in opinion were resolved by consensus.

Lesions with visually elevated uptake of ${ }^{68} \mathrm{Ga}-\mathrm{FAPI}$ in/around bones or joints compared to the surrounding background tissue were assessed. For each patient, the assessed dataset included:

- Number of Lesions Number of lesions was recorded, if the number in one patient was $>20$, the 20 lesions with the highest uptake were included.

- Locations of lesions The locations were identified as being located in joints or bones including the skull, alveolar ridges, sternum, spine, ribs, and appendicular skeleton.

- Classifications of lesions Each lesion was determined to be malignant or benign based on a comprehensive analysis of the patient's medical history and previous or subsequent conventional imaging data. Malignant lesions referred to primary bone cancer or bone metastases. Benign lesions were classified into five subgroups: osteofibrous dysplasia, degenerative bone disease, periodontitis, arthritis, and other inflammatory or trauma-related abnormalities (such as tendinitis, fracture or healing injuries). 
- SUVmax Maximum standardized uptake values (SUVmax) for ${ }^{68} \mathrm{Ga}$-FAPI-avid lesions were measured.

\section{Statistical analyses}

A commercial software package (SPSS 26.0, IBM Inc., Armonk NY, USA) was employed for statistical analysis. Continuous variables are expressed as mean \pm SD. Categorical variables are expressed as number and percentage. For quantitative data, normal distribution and homogeneity of variances were tested first. Quantitative data with non-normal distribution were compared using the Mann-Whitney U-test between two groups, and the Kruskal-Wallis $H$-test among the five groups. A $p$ value $<0.05$ was considered statistically significant.

\section{Results}

The image data of 129 cases were retrospectively reviewed. Elevated uptake of ${ }^{68} \mathrm{Ga}-\mathrm{FAPI}$ in/around the bone or joint was found in 82 cases (63.6\%) which were enrolled in this study. The patients ( 47 male and 35 female) had a mean age of 56.65 \pm 10.15 years (range, 26-80 years). A total of 73 patients underwent PET/MR and 9 patients underwent PET/CT. Table 1 presents the general information of the enrolled patients.

Table 1

General information of the enrolled patients

\begin{tabular}{|lll|}
\hline Characteristics & No. & $\%$ \\
\hline Gender & & \\
\hline Male & 47 & $57.3 \%$ \\
\hline Female & 35 & $42.7 \%$ \\
\hline Age $(y$, mean \pm SD, range) & $56.65 \pm 10.15 \mathrm{y},(26-80 \mathrm{y})$ \\
\hline Reasons of PET imaging & & \\
\hline Malignant tumor & 70 & $85.4 \%$ \\
\hline Fibrosis related diseases & 12 & $14.6 \%$ \\
\hline Scanner & & \\
\hline PET/MR & 73 & $89.0 \%$ \\
\hline PET/CT & 9 & $11.0 \%$ \\
\hline
\end{tabular}

In 82 cases, a total of $295{ }^{68} \mathrm{Ga}-\mathrm{FAPI}$-avid bone/joint lesions were identified, including 94 malignant bone lesions in 15 cases and 201 benign bone/joint lesions in 76 cases, of which 9 cases had both malignant and benign lesions (Fig. 1 and Fig. 2). All malignant lesions were diagnosed as bone metastases. The benign diagnoses were as follows: osteofibrous dysplasia (n $=13,4.4 \%)$, degenerative bone disease $(n=48,16.3 \%)$, periodontitis $(n=33,11.2 \%)$, arthritis $(n=56,19.0 \%)$ and other inflammatory or trauma-related lesions ( $n=51,17.3 \%)$.

As shown in Fig. 3, the mean SUVmax of bone metastases was significantly higher than that of benign diseases $(7.14 \pm 4.33$ vs. $3.57 \pm 1.60, p<0.0001$ ), but overlap occurred in some cases. Two representative cases (Fig. 1 and Fig. 2 ) had both bone metastases and benign lesions, which showed similar ${ }^{68} \mathrm{Ga}$-FAPI uptake. The differences in SUVmax among subgroups of benign disease were significant $(p<0.0001)$. The uptake of ${ }^{68} \mathrm{Ga}$-FAPI in periodontitis $(4.45 \pm 1.17)$ was much higher than that in other entities. However, paired comparisons of SUVmax in osteofibrous dysplasia ( $3.48 \pm 1.56)$, degenerative bone disease ( $3.53 \pm 1.68)$, arthritis $(3.24 \pm 1.48)$, and other inflammatory or trauma-related lesions $(3.41 \pm 1.76)$ showed no significant differences. 
Table 2 lists the detected lesion numbers. Most cases (62/82, 75.6\%) had 1-3 positive lesions in/around the bones and joints, and $34.2 \%(28 / 82)$ had only a single lesion. While $24.4 \%$ cases had $>3$ lesions, with $>20$ positive lesions presenting in two cases. Among the cases with metastases, $46.7 \%$ had $1-3$ bone metastases, and the remaining $53.3 \%$ of cases had $>3$ bone metastases. In cases of benign bone/joint diseases, $81.5 \%$ of cases had $1-3$ lesions, and $19.5 \%$ had $>3$ lesions.

Table 2

Distribution of number of lesions and percentage

\begin{tabular}{|lllllll|}
\hline \multirow{2}{*}{ No. of lesions } & All lesions & \multicolumn{3}{l}{ Benign diseases } & \multicolumn{2}{l|}{ Metastases } \\
\cline { 2 - 6 } & Frequencies & Percentage & Frequencies & Percentage & Frequencies & Percentage \\
\hline 1 & 28 & 34.1 & 28 & 36.8 & 4 & 26.7 \\
\hline 2 & 21 & 25.6 & 20 & 26.3 & 2 & 13.3 \\
\hline 3 & 13 & 15.9 & 14 & 18.4 & 1 & 6.7 \\
\hline 4 & 2 & 2.4 & 5 & 6.6 & 1 & 6.7 \\
\hline 5 & 2 & 2.4 & 3 & 3.9 & 2 & 13.3 \\
\hline 6 & 5 & 6.1 & 3 & 3.9 & 1 & 6.7 \\
\hline 7 & 1 & 1.2 & 0 & 0 & 0 & 0 \\
\hline 8 & 1 & 1.2 & 0 & 0 & 0 & 0 \\
\hline 9 & 2 & 2.4 & 0 & 0 & 1 & 6.7 \\
\hline 10 & 3 & 3.7 & 2 & 2.6 & 0 & 0 \\
\hline 16 & 0 & 0 & 0 & 0 & 1 & 6.7 \\
\hline 17 & 1 & 1.2 & 1 & 1.3 & 0 & 0 \\
\hline 18 & 1 & 1.2 & 0 & 0 & 0 & 0 \\
\hline 19 & 0 & 0 & 0 & 0 & 1 & 6.7 \\
\hline 20 & 2 & 100.0 & 76 & 100.0 & 15 & 100.0 \\
\hline Total & 2.4 & 0 & & & 1 \\
\hline
\end{tabular}

Table 3 summarises the distributions of different types of diseases. Vertebral bodies and posterior elements were the most frequently $(n=82,27.8 \%)$ involved sites. The shoulder joint $(n=45,15.3 \%)$ was the second most common site, followed by the alveolar ridge $(n=33,11.2 \%)$, the pelvis $(n=31,10.5 \%)$, ribs $(n=27,9.2 \%)$ and clavicle $(n=24,8.1 \%)$. 
Locations of each type of diseases

\begin{tabular}{|c|c|c|c|c|c|c|c|c|}
\hline \multirow[t]{2}{*}{ Locations } & \multirow{2}{*}{$\begin{array}{l}\text { No. of } \\
\text { patients } \\
\text { (\%) }\end{array}$} & \multirow{2}{*}{$\begin{array}{l}\text { No. of } \\
\text { lesions } \\
\text { (\%) }\end{array}$} & \multicolumn{3}{|c|}{ Benign Lesions, n (\%) } & \multirow[b]{2}{*}{ Periodontitis } & \multirow[b]{2}{*}{ Arthritis } & \multirow[b]{2}{*}{ Others* } \\
\hline & & & Metastases & $\begin{array}{l}\text { Osteofibrous } \\
\text { dysplasia }\end{array}$ & $\begin{array}{l}\text { Degenerative } \\
\text { bone } \\
\text { disease }\end{array}$ & & & \\
\hline Skull & $5(6.1)$ & $5(1.7)$ & $3(3.2)$ & $1(7.7)$ & & & & $1(2.0)$ \\
\hline $\begin{array}{l}\text { Alveolar } \\
\text { ridge }\end{array}$ & $\begin{array}{l}25 \\
(30.5)\end{array}$ & $\begin{array}{l}33 \\
(11.2)\end{array}$ & & & & $33(100.0)$ & & \\
\hline $\begin{array}{l}\text { Sternal } \\
\text { angle/ } \\
\text { Sternocostal } \\
\text { joint }\end{array}$ & $3(3.7)$ & $6(2.0)$ & & & & & & $\begin{array}{l}6 \\
(11.8)\end{array}$ \\
\hline Sternum & $1(1.2)$ & $2(0.7)$ & $2(2.1)$ & & & & & \\
\hline \multicolumn{9}{|l|}{ Spine } \\
\hline $\begin{array}{l}\text { Vertebral } \\
\text { body and } \\
\text { posterior } \\
\text { element }\end{array}$ & $\begin{array}{l}30 \\
(36.6)\end{array}$ & $\begin{array}{l}82 \\
(27.8)\end{array}$ & $40(42.6)$ & $1(7.7)$ & $39(81.3)$ & & & $2(3.9)$ \\
\hline $\begin{array}{l}\text { Intervertebral } \\
\text { disc }\end{array}$ & $5(6.1)$ & $6(2.0)$ & & & $4(8.3)$ & & & $2(3.9)$ \\
\hline $\begin{array}{l}\text { Spinal facet } \\
\text { joint }\end{array}$ & $1(1.2)$ & $9(3.1)$ & & & & & & $\begin{array}{l}9 \\
(17.6)\end{array}$ \\
\hline Ribs & $\begin{array}{l}12 \\
(14.6)\end{array}$ & $\begin{array}{l}27 \\
(9.2)\end{array}$ & $20(21.3)$ & $4(30.8)$ & & & & $3(5.9)$ \\
\hline \multicolumn{9}{|l|}{$\begin{array}{l}\text { Appendicular } \\
\text { skeleton and } \\
\text { joint }\end{array}$} \\
\hline Clavicle & $\begin{array}{l}15 \\
(18.3)\end{array}$ & $\begin{array}{l}24 \\
(8.1)\end{array}$ & $2(2.1)$ & $1(7.7)$ & & & & $\begin{array}{l}21 \\
(41.2)\end{array}$ \\
\hline Scapula & $1(1.2)$ & $1(0.3)$ & $1(1.1)$ & & & & & \\
\hline Pelvis & $\begin{array}{l}19 \\
(23.2)\end{array}$ & $\begin{array}{l}31 \\
(10.5)\end{array}$ & $24(25.5)$ & $2(15.4)$ & $4(8.3)$ & & & $1(2.0)$ \\
\hline $\begin{array}{l}\text { Tendon } \\
\text { attachment } \\
\text { in ischial } \\
\text { tuberosity }\end{array}$ & $4(4.9)$ & $6(2.0)$ & & & & & & $\begin{array}{l}6 \\
(11.8)\end{array}$ \\
\hline Humerus & $2(2.4)$ & $2(0.7)$ & & $1(7.7)$ & $1(2.1)$ & & & \\
\hline Femur & $5(6.1)$ & $5(1.7)$ & $2(2.1)$ & $3(23.1)$ & & & & \\
\hline $\begin{array}{l}\text { Shoulder } \\
\text { joint }\end{array}$ & $\begin{array}{l}32 \\
(39.0)\end{array}$ & $\begin{array}{l}45 \\
(15.3)\end{array}$ & & & & & $\begin{array}{l}45 \\
(80.4)\end{array}$ & \\
\hline Hip joint & $8(9.8)$ & $\begin{array}{l}11 \\
(3.7)\end{array}$ & & & & & $\begin{array}{l}11 \\
(19.6)\end{array}$ & \\
\hline Total No. & 82 & 295 & $94(31.9)$ & $13(4.4)$ & $48(16.3)$ & $33(11.2)$ & $\begin{array}{l}12 \\
56(19.0)\end{array}$ & $\begin{array}{l}51 \\
(17.3)\end{array}$ \\
\hline
\end{tabular}


Bone metastases were mainly located in the vertebral bodies and posterior elements $(n=40,42.6 \%)$, pelvis $(n=24,25.5 \%)$, ribs $(n=20,21.3 \%)$, with the rest distributed among the skull $(n=3,3.2 \%)$, sternum $(n=2,2.1 \%)$, clavicle $(n=2,2.1 \%)$, scapula $(n=1,1.1 \%)$, and femur $(n=2,2.1 \%)$. Figure 1 and Fig. 2 show two typical cases with multiple bone metastases (red arrows).

For the subgroup of benign diseases, osteofibrous dysplasia occurred at many sites, including the skull, clavicle, ribs, vertebral bodies, pelvis, and long bones, featuring ground-glass opacities with well-defined borders and intact overlying bone on CT images, and these lesions had not changed significantly for years (Fig. 1d and Fig. 4). Elevated uptake in the alveolar ridges was diagnosed as periodontitis (Fig. 5). The majority of degenerative bone diseases occurred in the spine, usually at the edge of the vertebral body, including osteodysgenesis, osteophyte and schmorl's node ( $n=39,81.3 \%$, Fig. 2d; Fig. 6d) and intervertebral discs $(n=4,8.3 \%)$. The most representative arthritis occurred in the shoulder $(n=45,80.4 \%$, Fig. $1 b$; Fig. $6 b)$ and hip ( $n=11,19.6 \%$, Fig. 6e). Other inflammatory or trauma-related abnormalities mainly appeared at the acromial or sternal end of clavicle ( $n=21,41.2 \%)$, spinal facet joints $(n=9,17.6 \%$, Fig. $6 c)$, sternal angle / sternocostal joint $(n=6,11.8 \%)$, and tendon attachment in ischial tuberosities ( $n=6,11.8 \%$, Fig. 1c). Figure 6 presents one representative case with 17 foci in bones and joints, all diagnosed as benign entities. One case showed healed fractures in three consecutive ribs (Fig. 7).

In areas with coexisting malignant and benign lesions, the proportion of the diseases was different. In the pelvis and ribs, malignant foci predominated $(25 / 31,20 / 27)$. In vertebral bodies and posterior elements (malignant $n=40$; benign $n=42$ ), skull (malignant $n=3$; benign $n=2$ ) and femur (malignant $n=2$; benign $n=3$ ), the ratios were closer to 1 , which suggests that more attention should be paid to distinguishing between benign and malignant in these sites.

\section{Discussion}

In this study, we found that ${ }^{68} \mathrm{Ga}$-FAPI is taken up by both benign and metastatic foci in bone as well as by foci of joint disease. The periodontitis, spinal degenerative disease, arthritis, and tendonitis were easily diagnosed due to their specific locations. Lesions involving the skull, ribs, vertebral bodies, pelvis, and long bones could be benign or malignant. Although the uptakes in metastatic lesions had higher SUVmax values than those of benign lesions, further evaluation of bone lesions is necessary because of overlap.

In healthy adult tissues, no or only insignificant levels of the FAP protein are detected [30], giving ${ }^{68} \mathrm{Ga}-\mathrm{FAPI}-04$ the advantage of low physiological uptake in normal tissues and is characterized by prolonged tumor retention time, which makes it an ideal tracer for tumor imaging [7]. Tumor diagnosis and follow-up is the most important direction of ${ }^{68} \mathrm{Ga}-\mathrm{FAPI}$ research [3]. Increased ${ }^{68} \mathrm{Ga}$-FAPI uptake in primary tumors and many metastases occurs in various cancers, including bone metastases [9, $10,31,32$. However, in this study, we found that $68.1 \%$ of foci with elevated uptake in the bones and joints were in benign disease entities. This suggested that differential diagnosis is very important. We found that the characteristics of metastases include higher ${ }^{68} \mathrm{Ga}$-FAPI uptake, a higher number of foci and predominant occurrence in the axial skeleton. Based on 201 benign lesions in our results, the features of ${ }^{68} \mathrm{Ga}$-FAPI-positive benign diseases included more solitary lesions, lower uptake, and specific locations, including the shoulder and hip joint, alveolar ridge, acromial or sternal end of clavicle, spinal facet joint, sternal angle / sternocostal joint, tendon attachment in ischial tuberosity.

The expression of FAP in some benign diseases and in normal tissues during remodelling by stromal cells and mesenchymal stem cells during embryogenesis, wound healing, fibrotic reactions, and inflammatory conditions such as arthritis, atherosclerotic plaques and fibrosis [30], explains the uptake of ${ }^{68} \mathrm{Ga}-\mathrm{FAPI}$ in these entities. FAP is also expressed by rheumatoid myofibroblast-like synoviocytes [33]. FAPI uptake in some benign tumors (including angiomyolipoma and solitary fibrous tumor) [14-16] and non-neoplastic benign diseases (including IgG4-related diseases, cardiovascular disease, and idiopathic retroperitoneal fibrosis) with active fibrosis is known [17-21], and physiological uptake in some organs like breast and uterus are common, which suggests that radiolabelled FAPI is a relatively nonspecific tumor tracer. To date, reports on FAPI uptake in benign bone and joint benign diseases have been limited. 
In this study, 13 bone foci were diagnosed as osteofibrous dysplasia, which constitutes $5 \%$ of all benign bone lesions [34] and generated by replacement of portions of normal bone by focal fibrous proliferation, which results in disorganised trabecular formation [35]. On CT, it usually has typical features of ground-glass density and cystic low-density areas [36]. Elevated ${ }^{68} \mathrm{Ga}$ FAPI uptake was observed in the alveolar ridges, which may be related to the fibroblasts centrally involved in the reformation of periodontal tissue structures in the healing response [37]. Our results revealed SUVmax of periodontitis is much higher than that in other benign conditions, which likely indicates more active fibroblasts. High uptake at both ends of clavicle and tendon attachment in ischial tuberosities could be explained as inflammation or tendonitis because FAP is highly expressed in tissue repair. Similarly, both ${ }^{68} \mathrm{Ga}$-FAPI-02 and ${ }^{68} \mathrm{Ga}$-FAPI-04 have been reported to have uptake in locations of wound healing after surgical intervention [1]. In our study, 48 degenerative bone lesions revealed high uptake. A recent report demonstrated ${ }^{68} \mathrm{Ga}-$ FAPI accumulation in degenerative osteophytes [38]. The ${ }^{68} \mathrm{Ga}$-FAPI uptake by degenerative osteophytes may be a result of the presence of fibroblasts in early osteophytes $[39,40]$. FAPa is reported to be expressed in human and rat degenerative intervertebral disc disease [28]. The corresponding CT or MR images revealed the specific location of the disease, which made it easier to distinguish degenerative disease from bone metastases.

Although bone metastases had higher ${ }^{68} \mathrm{Ga}$-FAPI uptake than benign bone and joint diseases, our results revealed that uptake by malignant and benign lesions had some overlap (Fig. 3). As shown in two cases in Fig. 1 and Fig. 2, which had both bone metastases and benign lesions with almost the same intensity of ${ }^{68} \mathrm{Ga}$-FAPI uptake, again demonstrated its lack of specificity for malignancy in bone. Except for some diseases with special locations and characteristic CT and MR manifestations, it is very important to distinguish bone metastases from benign bone disease in non-specific locations in patients with a malignancy. Comprehensive careful analysis with medical history and conventional imaging remains essential for diagnostic accuracy.

There are several limitations in this study. First, the types of bone diseases included in this study were limited. No primary bone malignancy was included. Only a few kinds of benign bone diseases were noticed and analysed. Second, no pathological evidence was obtained. The final diagnosis was based on comprehensive analysis, including clinical data, imaging features, and related imaging examinations. Third, this was an observational study with a limited number of patients, and the original purpose of the clinical researches was not to explore the diagnostic value of ${ }^{68} \mathrm{Ga}$-FAPI PET in bone and joint lesions. In spite of these limitations, we found that both benign and malignant bone lesions could uptake ${ }^{68} \mathrm{Ga}-\mathrm{FAPI}$, which suggests that we should pay attention to the differential diagnosis for malignant bone lesions, especially for the single lesion, because the diagnosis of bone metastasis may upstage the disease and change treatment.

\section{Conclusion}

${ }^{68} \mathrm{Ga}-\mathrm{FAPI}$ can be taken up by both bone metastases and benign osteoarticular lesions. The uptake in bone metastases was higher than that of benign diseases in bones and joints, but there was overlap. Abnormal osseous ${ }^{68} \mathrm{Ga}$-FAPI uptake should be carefully assessed in patients with malignant tumors, especially in solitary bone lesions, to avoid misdiagnosis.

\section{Declarations}

\section{Acknowledgement}

We thank Libby Cone, MD, MA, from Liwen Bianji, Edanz Group China (www.liwenbianji.cn/ac) for editing a draft of this manuscript.

\section{Funding}

This work was supported in part by the Key Project of National Natural Science Foundation of China (No. 82030052).

\section{Conflicts of interest}


The authors declare that they have no conflict of interest.

\section{Ethics approval}

This article does not contain any experiments with animals. All procedures involving human participants were carried out in accordance with the ethical standards of the institutional and/or national research committee and with the 1964 Helsinki Declaration and its later amendments or comparable ethical standards. This study was approved by the Clinical Research Ethics Committee of Union Hospital, Tongji Medical College, Huazhong University of Science and Technology (NO. 20200290 and NO. 20200507).

\section{Consent to participate}

Informed consent was obtained from all individual participants included in this study.

Trial registration: NCT04554719, NCT04605939. Registered September 8, 2020 and October, 25, 2020 - retrospectively registered, http://clinicaltrails.gov/show/ NCT04554719; http://clinicaltrails.gov/show/ NCT04605939

\section{References}

1. Lindner T, Loktev A, Altmann A, Giesel F, Kratochwil C, Debus J, et al. Development of Quinoline-Based Theranostic Ligands for the Targeting of Fibroblast Activation Protein. Journal of nuclear medicine: official publication. Society of Nuclear Medicine. 2018;59:1415-22. doi:10.2967/jnumed.118.210443.

2. Mersmann M, Schmidt A, Rippmann JF, Wüest T, Brocks B, Rettig WJ, et al. Human antibody derivatives against the fibroblast activation protein for tumor stroma targeting of carcinomas. Int J Cancer. 2001;92:240-8. doi:10.1002/10970215(200102)9999:9999<::aid-ijc1170>3.0.co;2-u.

3. Calais JFAP. The Next Billion Dollar Nuclear Theranostics Target? J Nucl Med. 2020;61:163-5. doi:10.2967/jnumed.119.241232.

4. Lindner T, Altmann A, Krämer S, Kleist C, Loktev A, Kratochwil C, et al. Design and Development of (99m)Tc-Labeled FAPI Tracers for SPECT Imaging and (188)Re Therapy. Journal of nuclear medicine: official publication. Society of Nuclear Medicine. 2020;61:1507-13. doi:10.2967/jnumed.119.239731.

5. Toms J, Kogler J, Maschauer S, Daniel C, Schmidkonz C, Kuwert T, et al. Targeting Fibroblast Activation Protein: Radiosynthesis and Preclinical Evaluation of an (18)F-labeled FAP Inhibitor. Journal of nuclear medicine: official publication, Society of Nuclear Medicine. 2020. doi:10.2967/jnumed.120.242958.

6. Watabe T, Liu Y, Kaneda-Nakashima K, Shirakami Y, Lindner T, Ooe K, et al. Theranostics targeting fibroblast activation protein in the tumor stroma: (64)Cu and (225)Ac labelled FAPI-04 in pancreatic cancer xenograft mouse models. Journal of nuclear medicine: official publication Society of Nuclear Medicine. 2019. doi:10.2967/jnumed.119.233122.

7. Giesel FL, Kratochwil C, Lindner T, Marschalek MM, Loktev A, Lehnert W, et al. (68)Ga-FAPI PET/CT: Biodistribution and Preliminary Dosimetry Estimate of 2 DOTA-Containing FAP-Targeting Agents in Patients with Various Cancers. Journal of nuclear medicine: official publication. Society of Nuclear Medicine. 2019;60:386-92. doi:10.2967/jnumed.118.215913.

8. Loktev A, Lindner T, Mier W, Debus J, Altmann A, Jager D, et al. A Tumor-Imaging Method Targeting Cancer-Associated Fibroblasts. Journal of nuclear medicine: official publication. Society of Nuclear Medicine. 2018;59:1423-9. doi:10.2967/jnumed.118.210435.

9. Kratochwil C, Flechsig P, Lindner T, Abderrahim L, Altmann A, Mier W, et al. (68)Ga-FAPI PET/CT: Tracer Uptake in 28 Different Kinds of Cancer. Journal of nuclear medicine: official publication. Society of Nuclear Medicine. 2019;60:801-5. doi:10.2967/jnumed.119.227967.

10. Pang Y, Zhao L, Luo Z, Hao B, Wu H, Lin Q, et al. Comparison of (68)Ga-FAPI and (18)F-FDG Uptake in Gastric, Duodenal, and Colorectal Cancers. Radiology. 2021;298:393-402. doi:10.1148/radiol.2020203275. 
11. Chen H, Pang Y, Wu J, Zhao L, Hao B, Wu J, et al. Comparison of [(68)Ga]Ga-DOTA-FAPI-04 and [(18)F] FDG PET/CT for the diagnosis of primary and metastatic lesions in patients with various types of cancer. Eur J Nucl Med Mol Imaging. 2020;47:1820-32. doi:10.1007/s00259-020-04769-z.

12. Sharma P, Singh SS, Gayana S. Fibroblast Activation Protein Inhibitor PET/CT: A Promising Molecular Imaging Tool. Clinical nuclear medicine. 2021;46:e141-e50. doi:10.1097/rlu.0000000000003489.

13. Zhuravlev F. Theranostic radiopharmaceuticals targeting cancer-associated fibroblasts. Current radiopharmaceuticals. 2020. doi:10.2174/1874471013666201224114148.

14. Qin C, Gai Y, Liu Q, Shao F, Lan X. Elevated 68Ga-FAPI Accumulation in a Recurrent Angiomyolipoma. Clinical nuclear medicine. 2020;45:1034-5. doi:10.1097/rlu.0000000000003345.

15. Wang R, Liu Q, Sui H, Zhang M, Zhu Z, Cui R. (68)Ga-FAPI outperforms (18)F-FDG PET/CT in identifying solitary fibrous tumor. Eur J Nucl Med Mol Imaging. 2021. doi:10.1007/s00259-020-05181-3.

16. Liu H, Liu L, Chen L, Zhao Y, Zhang W, Cai L, et al. [(68)Ga]Ga-DOTA-FAPI-04 PET/CT imaging of benign pulmonary solitary fibrous tumour. Eur J Nucl Med Mol Imaging. 2021. doi:10.1007/s00259-021-05199-1.

17. Schmidkonz C, Rauber S, Atzinger A, Agarwal R, Götz TI, Soare A, et al. Disentangling inflammatory from fibrotic disease activity by fibroblast activation protein imaging. Ann Rheum Dis. 2020;79:1485-91. doi:10.1136/annrheumdis-2020217408.

18. Heckmann MB, Reinhardt F, Finke D, Katus HA, Haberkorn U, Leuschner F, et al. Relationship Between Cardiac Fibroblast Activation Protein Activity by Positron Emission Tomography and Cardiovascular Disease. Circulation Cardiovascular imaging. 2020;13:e010628. doi:10.1161/circimaging.120.010628.

19. Pan Q, Luo Y, Zhang W. Idiopathic Retroperitoneal Fibrosis With Intense Uptake of 68Ga-Fibroblast Activation Protein Inhibitor and 18F-FDG. Clinical nuclear medicine. 2021;46:175-6. doi:10.1097/rlu.0000000000003402.

20. Zhou Y, He J, Chen Y. (68)Ga-FAPI PET/CT imaging in a patient with thyroiditis. Endocrine. 2021. doi:10.1007/s12020021-02605-4.

21. Luo Y, Pan Q, Yang H, Peng L, Zhang W, Li F. Fibroblast activation protein targeted PET/CT with (68)Ga-FAPI for imaging IgG4-related disease: comparison to (18)F-FDG PET/CT. Journal of nuclear medicine: official publication, Society of Nuclear Medicine. 2020. doi:10.2967/jnumed.120.244723.

22. Pang Y, Hao B, Shang Q, Sun L, Chen H. Comparison of 68Ga-FAPI and 18F-FDG PET/CT in a Patient With Cholangiocellular Carcinoma: A Case Report. Clinical nuclear medicine. 2020;45:566-7. doi:10.1097/rlu.0000000000003056.

23. Pang Y, Huang H, Fu L, Zhao L, Chen H. 68Ga-FAPI PET/CT Detects Gastric Signet-Ring Cell Carcinoma in a Patient Previously Treated for Prostate Cancer. Clinical nuclear medicine. 2020;45:632-5. doi:10.1097/rlu.0000000000003099.

24. Pang Y, Zhao L, Chen H. 68Ga-FAPI Outperforms 18F-FDG PET/CT in Identifying Bone Metastasis and Peritoneal Carcinomatosis in a Patient With Metastatic Breast Cancer. Clinical nuclear medicine. 2020;45:913-5. doi:10.1097/rlu.0000000000003263.

25. Xu T, Zhao Y, Ding H, Cai L, Zhou Z, Song Z, et al. [(68)Ga]Ga-DOTA-FAPI-04 PET/CT imaging in a case of prostate cancer with shoulder arthritis. Eur J Nucl Med Mol Imaging. 2020. doi:10.1007/s00259-020-05028-x.

26. Liu H, Wang Y, Zhang W, Cai L, Chen Y. Elevated [(68)Ga]Ga-DOTA-FAPI-04 activity in degenerative osteophyte in a patient with lung cancer. Eur J Nucl Med Mol Imaging. 2020. doi:10.1007/s00259-020-05090-5.

27. Tang YH, Yue ZS, Zheng WJ, Shen HF, Zeng LR, Hu ZQ, et al. 4-Phenylbutyric acid presents therapeutic effect on osteoarthritis via inhibiting cell apoptosis and inflammatory response induced by endoplasmic reticulum stress. Biotechnol Appl Chem. 2018;65:540-6. doi:10.1002/bab.1642.

28. Lv FJ, Peng Y, Lim FL, Sun Y, Lv M, Zhou L, et al. Matrix metalloproteinase 12 is an indicator of intervertebral disc degeneration co-expressed with fibrotic markers. Osteoarthritis cartilage. 2016;24:1826-36. doi:10.1016/j.joca.2016.05.012. 
29. Croft AP, Campos J, Jansen K, Turner JD, Marshall J, Attar M, et al. Distinct fibroblast subsets drive inflammation and damage in arthritis. Nature. 2019;570:246-51. doi:10.1038/s41586-019-1263-7.

30. Altmann A, Haberkorn U, Siveke J. The Latest Developments in Imaging of Fibroblast Activation Protein. Journal of nuclear medicine: official publication. Society of Nuclear Medicine. 2021;62:160-7. doi:10.2967/jnumed.120.244806.

31. Koerber SA, Staudinger F, Kratochwil C, Adeberg S, Haefner MF, Ungerechts G, et al. The role of FAPI-PET/CT for patients with malignancies of the lower gastrointestinal tract - first clinical experience. Journal of nuclear medicine: official publication Society of Nuclear Medicine. 2020. doi:10.2967/jnumed.119.237016.

32. Windisch P, Zwahlen DR, Koerber SA, Giesel FL, Debus J, Haberkorn U, et al. Clinical Results of Fibroblast Activation Protein (FAP) Specific PET and Implications for Radiotherapy Planning: Systematic Review Cancers (Basel). $2020 ; 12$. doi:10.3390/cancers12092629.

33. Bauer S, Jendro MC, Wadle A, Kleber S, Stenner F, Dinser R, et al. Fibroblast activation protein is expressed by rheumatoid myofibroblast-like synoviocytes. Arthritis research therapy. 2006;8:R171. doi:10.1186/ar2080.

34. DiCaprio MR, Enneking WF. Fibrous dysplasia. Pathophysiology, evaluation, and treatment. The Journal of bone joint surgery American volume. 2005;87:1848-64. doi:10.2106/jbjs.d.02942.

35. Bianco P, Kuznetsov SA, Riminucci M, Fisher LW, Spiegel AM, Robey PG. Reproduction of human fibrous dysplasia of bone in immunocompromised mice by transplanted mosaics of normal and Gsalpha-mutated skeletal progenitor cells. J Clin Investig. 1998;101:1737-44. doi:10.1172/jci2361.

36. Javaid MK, Boyce A, Appelman-Dijkstra N, Ong J, Defabianis P, Offiah A, et al. Best practice management guidelines for fibrous dysplasia/McCune-Albright syndrome: a consensus statement from the FD/MAS international consortium. Orphanet J Rare Dis. 2019;14:139. doi:10.1186/s13023-019-1102-9.

37. Smith PC, Martinez C, Martinez J, McCulloch CA. Role of Fibroblast Populations in Periodontal Wound Healing and Tissue Remodeling. Frontiers in physiology. 2019;10:270. doi:10.3389/fphys.2019.00270.

38. Dohi O, Ohtani H, Hatori M, Sato E, Hosaka M, Nagura H, et al. Histogenesis-specific expression of fibroblast activation protein and dipeptidylpeptidase-IV in human bone and soft tissue tumours. Histopathology. 2009;55:432-40. doi:10.1111/j.1365-2559.2009.03399.x.

39. Matyas JR, Sandell LJ, Adams ME. Gene expression of type II collagens in chondro-osteophytes in experimental osteoarthritis. Osteoarthritis cartilage. 1997;5:99-105. doi:10.1016/s1063-4584(97)80003-9.

40. van der Kraan PM, van den Berg WB. Osteophytes: relevance and biology. Osteoarthritis cartilage. 2007;15:237-44. doi:10.1016/j.joca.2006.11.006.

\section{Figures}




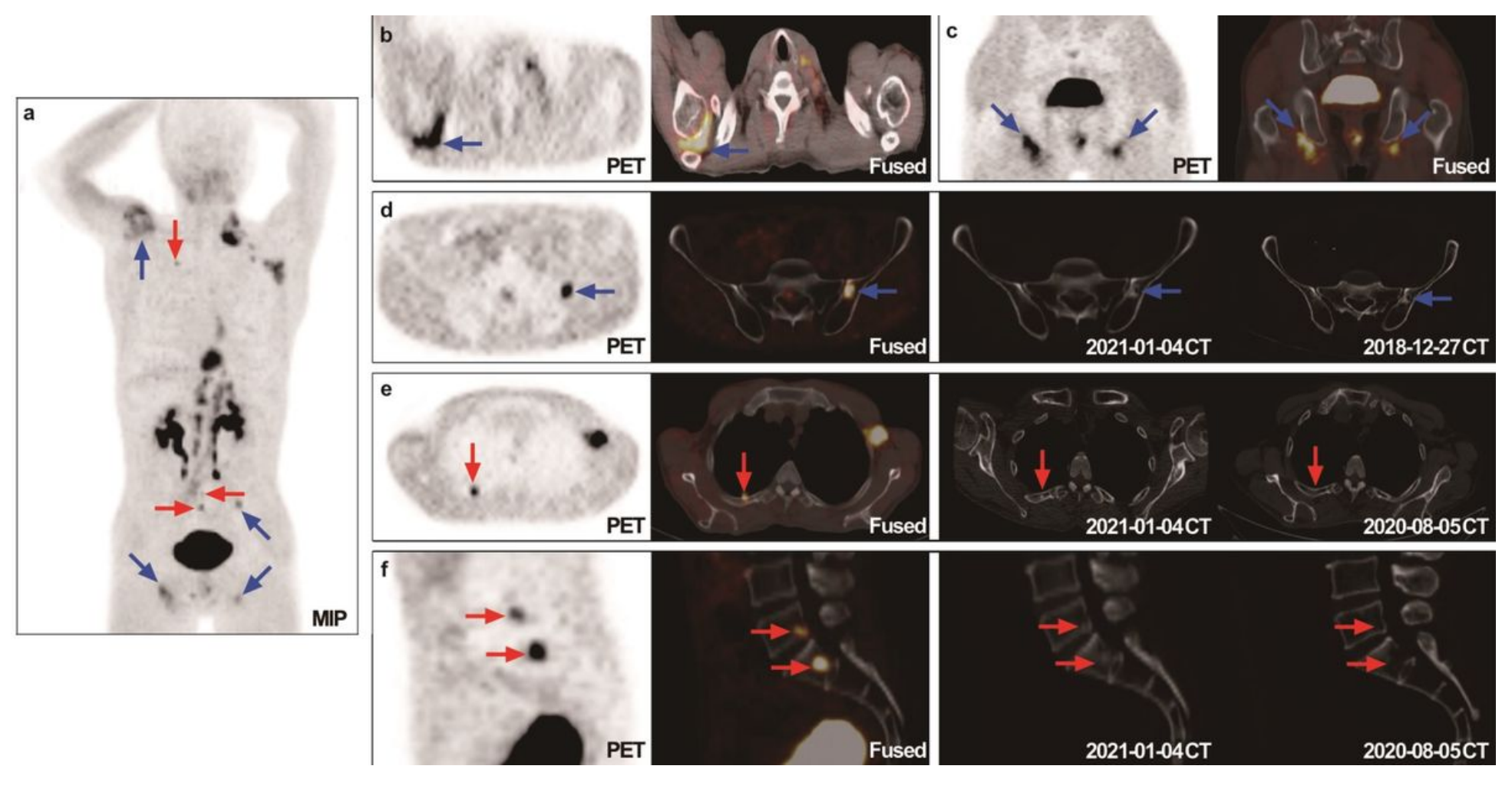

Figure 1

A 54-year-old man with a history of clinical stage III signet ring cell gastric adenocarcinoma underwent chemotherapy 3 times after radical operation. He underwent 68Ga-FAPI PET/CT for evaluation of the progress of disease. Cambered 68Ga-FAPI uptakes were clearly revealed in the right shoulder (SUVmax 8.3; b, blue arrows) and tendon attachment in bilateral ischial tuberosities (SUVmax 8.1; c, blue arrows), which were diagnosed as benign inflammatory lesions. A focus of elevated activity with SUVmax 5.5 in the left ilium was diagnosed as osteofibrous dysplasia because of characteristic ground-glass opacity with the low-density shadow which was the same as two years ago (d, blue arrows). Multiple aggregation of 68Ga-FAPI distributed in the ribs (e, red arrows, SUVmax 5.5) and the fifth lumbar vertebrae and the first sacrum (f, red arrows, SUVmax $3.5,7.6)$ with new emerging high-density shadows on CT images compared with the CT images 5 months ago, which were considered to be metastases. 


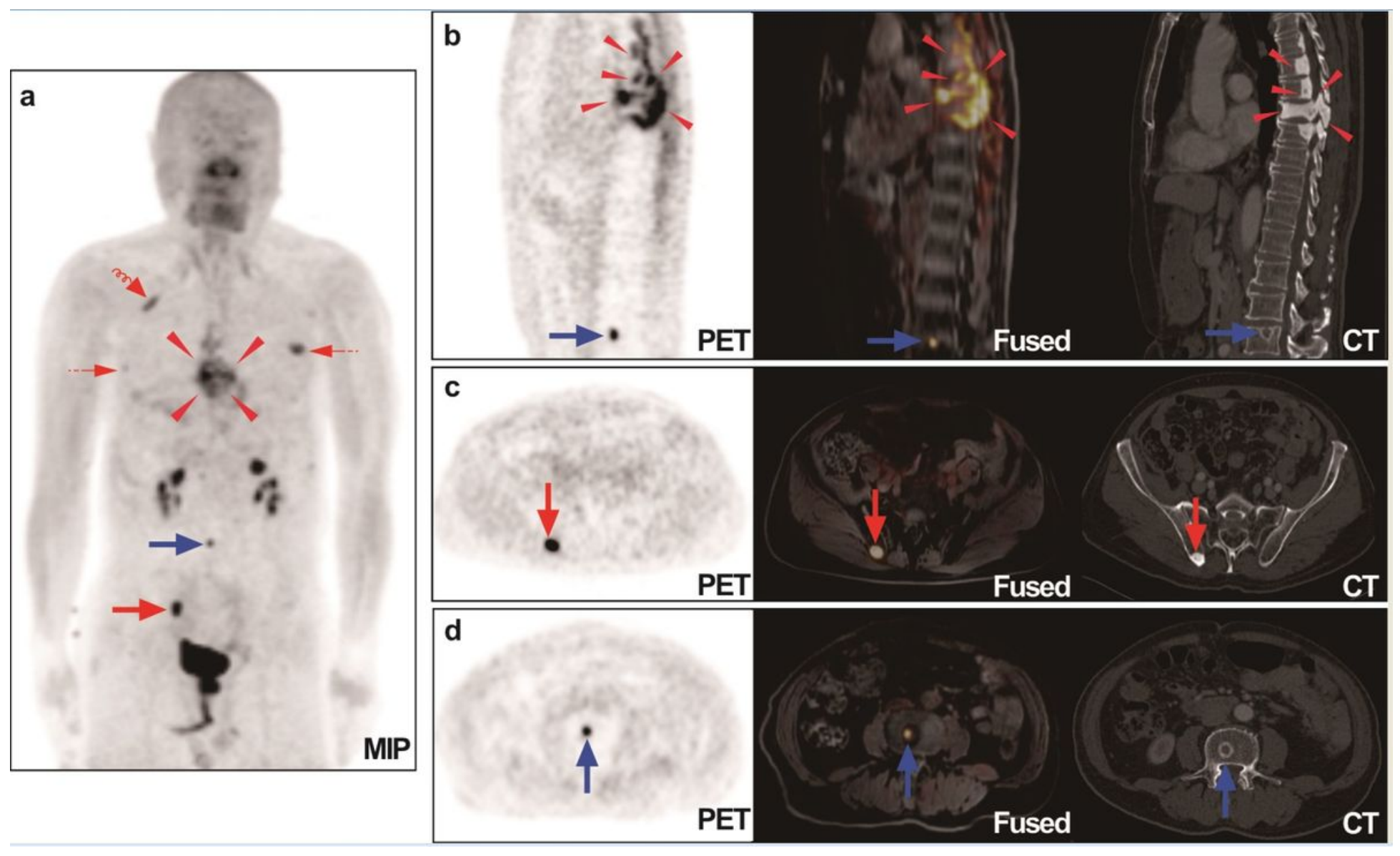

\section{Figure 2}

A 73-year-old man highly suspected of bone metastases performed 68Ga-FAPI PET/CT to detect the primary tumor. He was diagnosed as prostate cancer. The images revealed 16 bone metastases distributed in the ribs ( $\mathrm{a}$, thin red arrows), scapulae (a, curved red arrow), vertebral bodies and posterior elements (a \& b, red arrowheads) and pelvis (a \& c, red arrows), with SUVmax from 2.6 to 14.6, and most of which presented with hyperdense shadow on CT. Interestingly, a schmorl's node with accumulation of FAPI was observed on CT with the same signal characteristics as the adjacent disc and a thin rim of sclerosis at the margins (a, b \& d, blue arrows; SUVmax 9.2). 

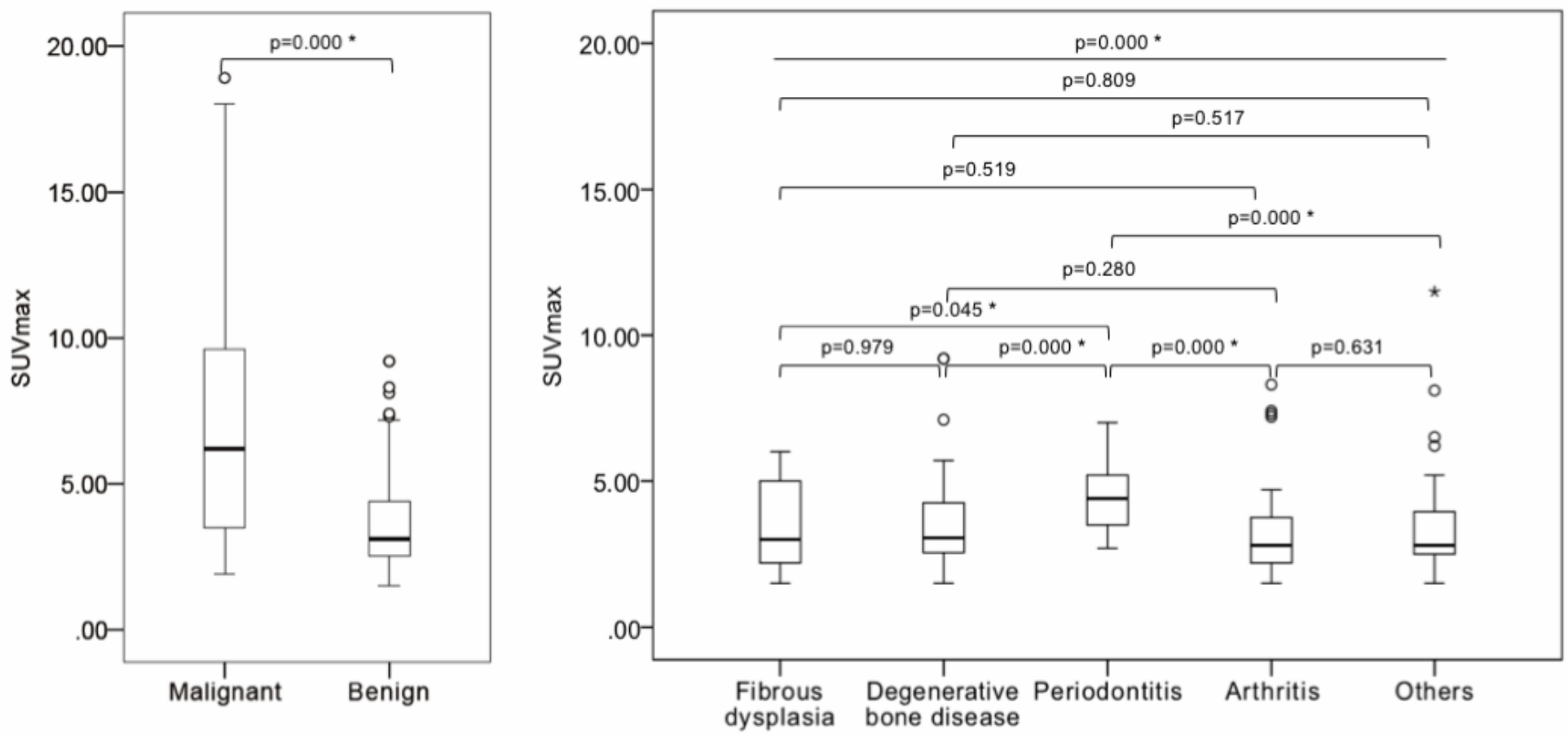

Figure 3

Boxplot of SUVmax in detected lesions and comparison in different groups

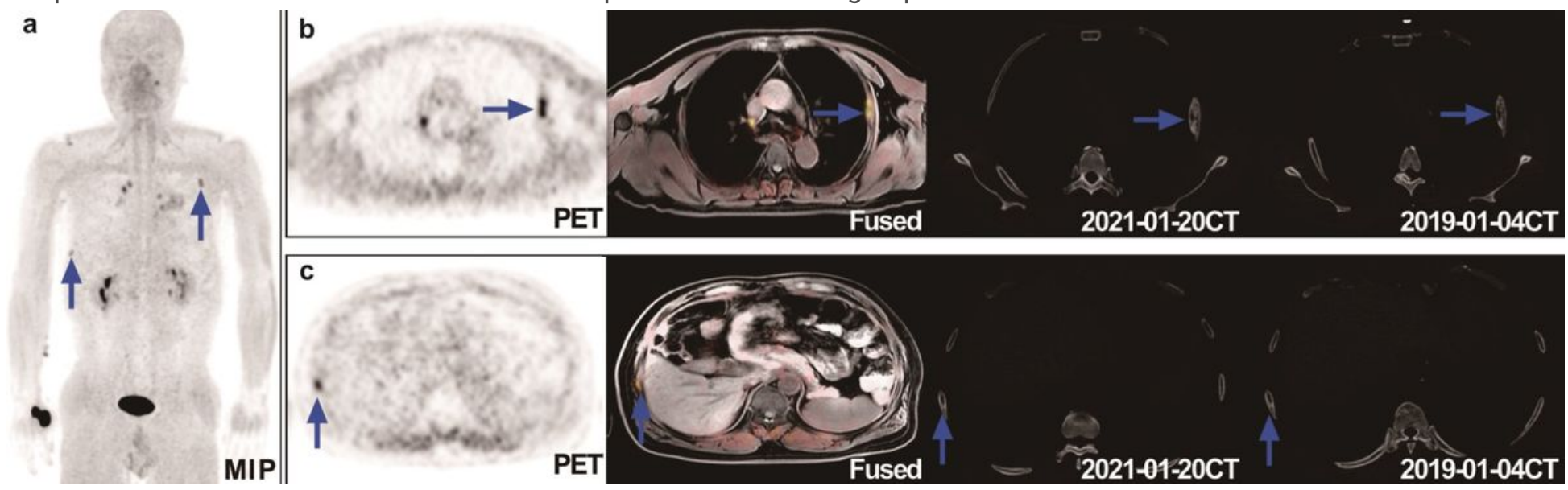

Figure 4

A 58-year-old male has performed 68Ga-FAPI PET/MR for detecting recurrence and metastases after 5-year comprehensive treatment of gastric cancer. Aggregation of 68Ga-FAPI on the left anterior fourth rib ( $\mathrm{a} \& \mathrm{~b}$, blue arrows) and the right ninth rib (a \& c, blue arrows) appeared with heterogeneous signals on MR (b \& c, blue arrows on Fused), corresponding to welldemarcated lesions with irregular ground-glass opacities and part of low-density shadow on the recent CT (b \& c, 2020-01-20 CT, blue arrows), same as CT two years ago (b \& c, 2019-01-4 CT, blue arrows). From the medical history and imaging findings, the two lesions were highly suspected as osteofibrous dysplasia of bone. 
a
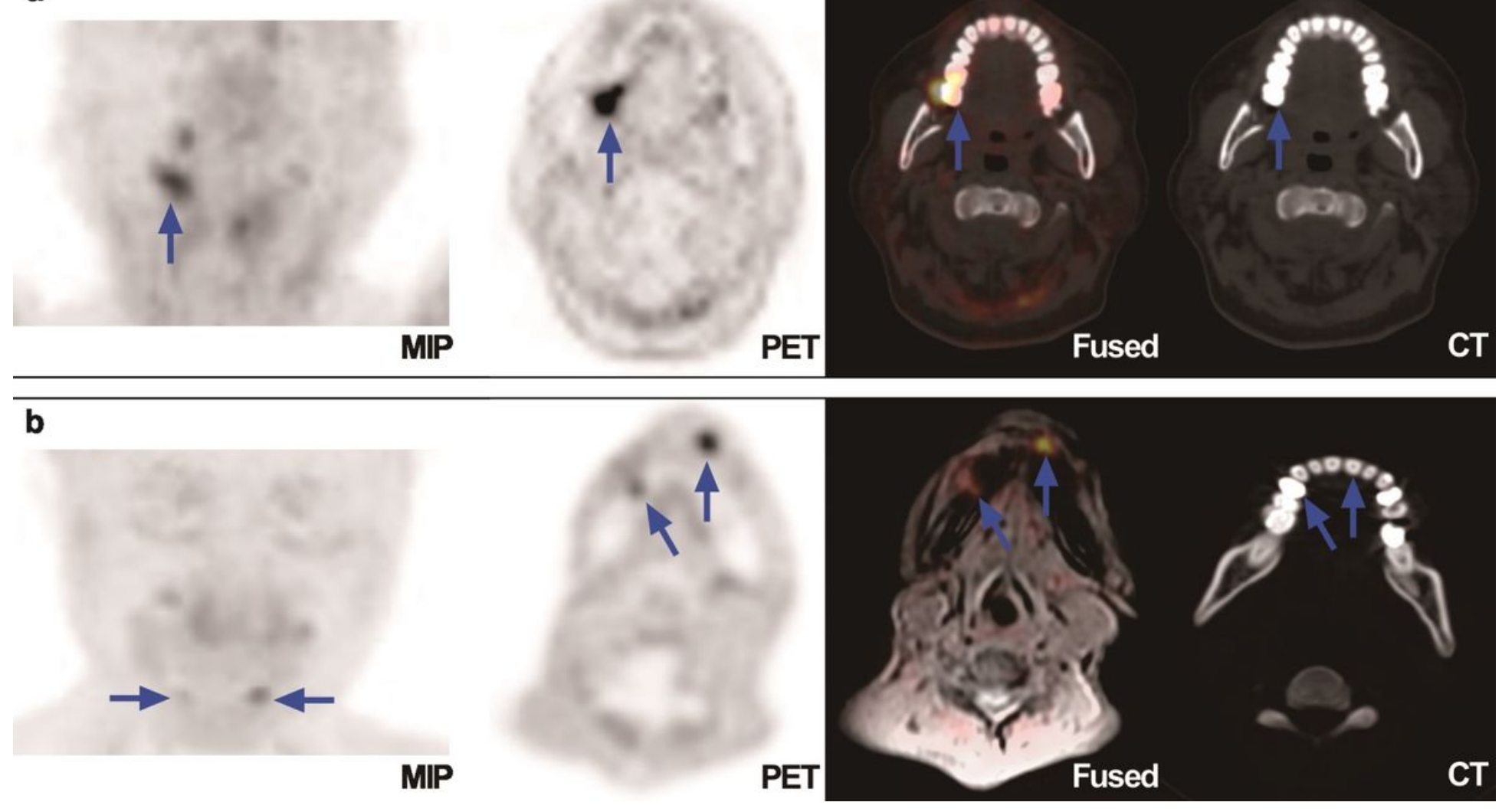

Figure 5

Accumulation of 68Ga-FAPI was presented on the right maxillary first and second molars (a, blue arrows) in a 48-year-old man with a history of dermatomyositis and interstitial pneumonia. A 54-year-old woman with a history of gastric cancer was also found elevated uptake of $68 \mathrm{Ga}-\mathrm{FAPI}$ in the mandibular central incisor and first premolar with partial denture (b, blue arrows). The lesions were diagnosed as periodontitis based on clinical symptoms of toothache and imaging findings.

a

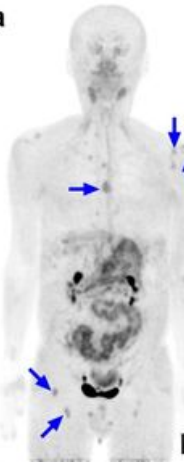

b

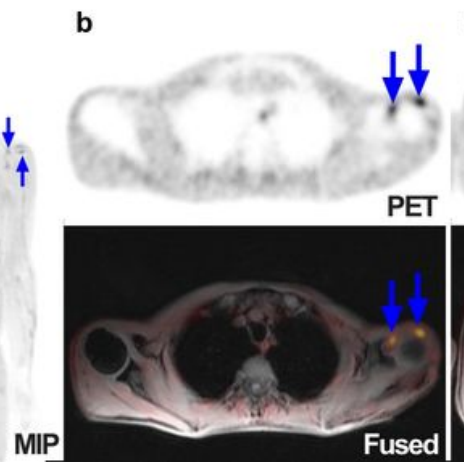

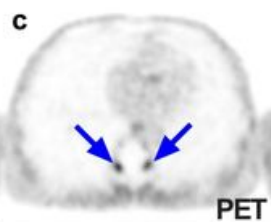

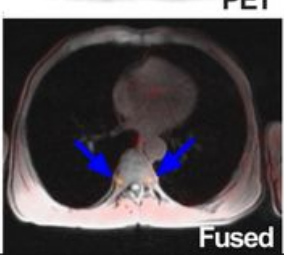

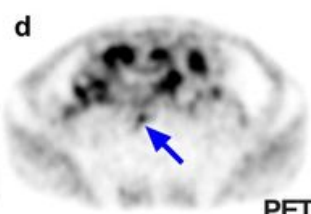

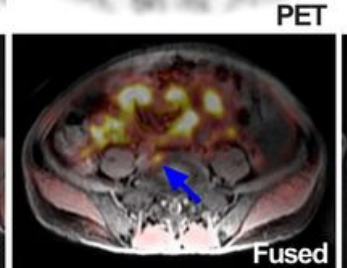

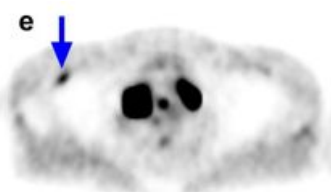

PET
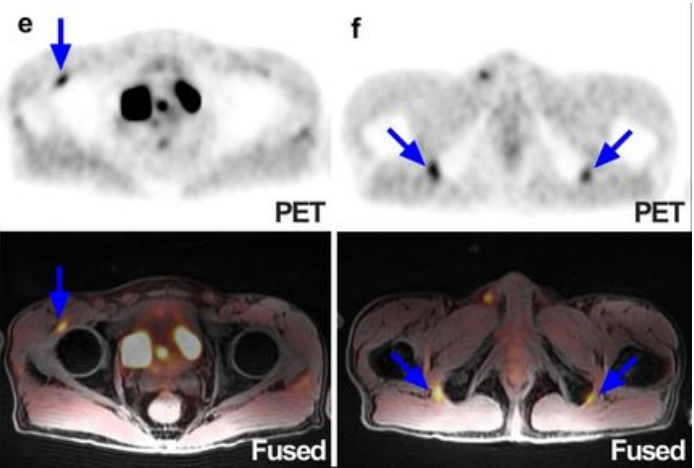

Figure 6

A 61-year-old male underwent 68Ga-FAPI PET/MR for detecting the primary lesion of unexplained ascites. Diffuse intense uptake was observed in the extensive thickened gastric wall, omentum and peritoneum (a), which suggested gastric cancer with celiac extensive metastases. Unexpectedly, elevated 68Ga-FAPI uptake appeared on 17 foci of bones and joints which were all diagnosed as benign, just around the left shoulder joint (b, blue arrows), edge of the vertebral body (c, blue arrows), facet joints (d, blue arrows), right femoral head (e, blue arrows), and tendon attachment in bilateral ischial tuberosities (f, blue arrows) with SUVmax of 3.3-4.2. MRI revealed no significant signal change. Lesions at the edge of vertebral body were diagnosed as retrogression, while the rest were considered to be inflammations such as arthritis and tendinitis. 


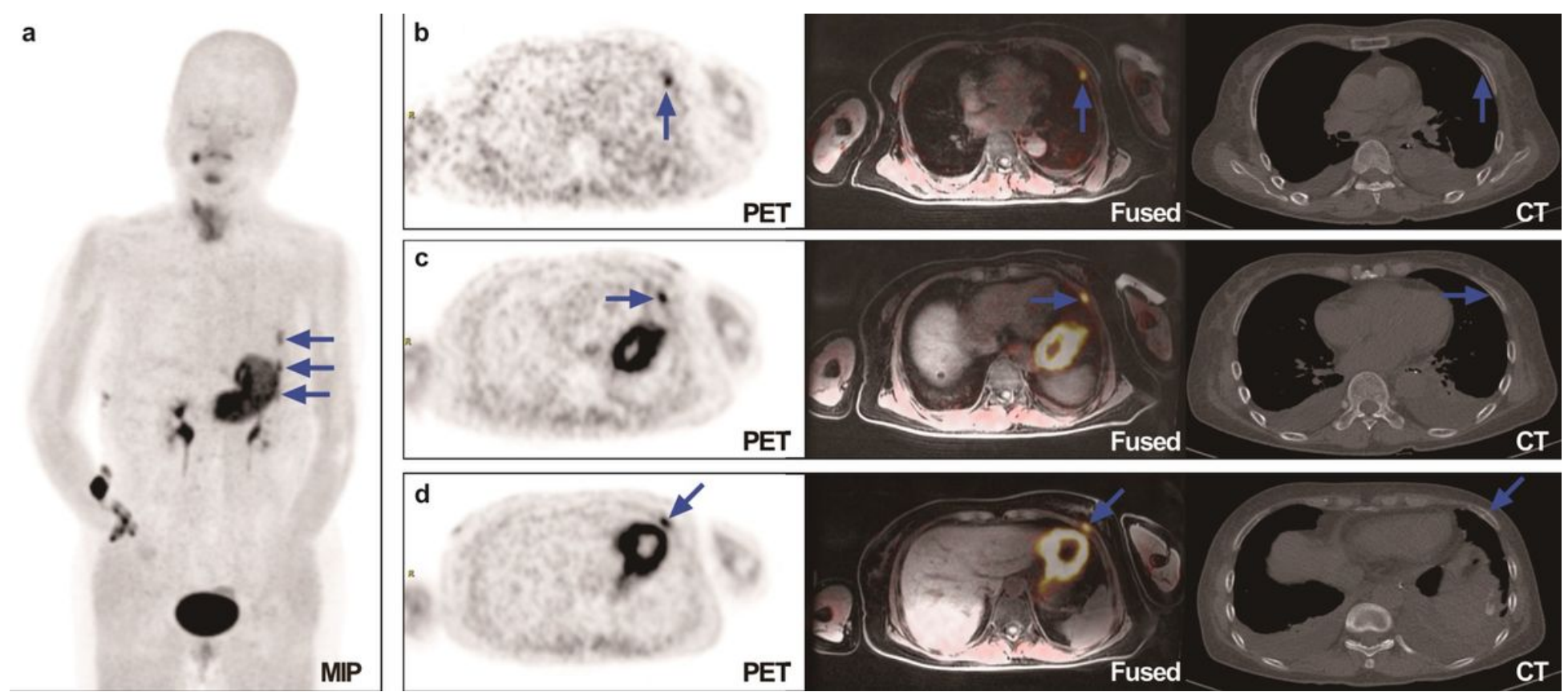

Figure 7

68Ga-FAPI PET revealed 3 foci accumulation in 3 contiguous ribs on the left with SUVmax 5.0-6.2 (a-d, blue arrows) in a 70year-old female with gastric cancer, which was diagnosed as fractures because of fracture lines were observed with increasing sclerosis and cortical thickening without relative displacement (b-d, blue arrows on CT). 